\title{
FutureJournal
}

\section{A Influência da Inteligência Emocional nas Competências Interpessoais do Gerente de Projetos e Sucesso no Gerenciamento de Projeto}

\author{
Luiz Fernando Lima \\ Mestrando em Gestão de Projetos pela Universidade Nove de Julho (UNINOVE), Brasil \\ luizlima.engenheiro@gmail.com
}

Filipe Quevedo-Silva

Doutor em Administração pela Universidade Nove de Julho (UNINOVE), Brasil

filquevedo@gmail.com

\section{RESUMO}

A inteligência emocional é um termo relativamente novo na área de gerenciamento de projetos, apesar de que, desde de sua origem, é estudada no ambiente de trabalho em geral. Contudo, nos últimos anos, o número de estudos com foco em inteligência emocional e gestão de projetos vem crescendo. Além disso, alguns autores têm buscado entender como se dá a relação entre as competências interpessoais do gerente de projetos e o sucesso no gerenciamento de projetos com o grau de inteligência emocional dos gestores. Neste artigo, o objetivo é construir proposições que relacionem a inteligência emocional com as competências interpessoais do gerente de projetos e o sucesso no gerenciamento de projeto. Para isso, foi feita uma revisão sistemática da literatura na temática. Com os resultados, espera-se fornecer subsídios teóricos para formulação de hipóteses que possam vir a ser testadas em futuros estudos empíricos.

PALAVRAS-CHAVE: Inteligência emocional. Gestão de projetos. Sucesso no gerenciamento de projetos. 


\section{FutureJournal}

The Influence of the Emotional Intelligence on Interpersonal Skills of Project Managers and Project Management Success

\section{ABSTRACT}

Emotional intelligence is a relatively new term in the project management area, although since its origin is studied in the general working environment. However, in recent years the number of studies focusing on emotional intelligence and project management is growing. In addition, some authors have sought to understand the relationship between the interpersonal skills of the project manager and success in project management to the degree of emotional intelligence of managers. This article aims to build propositions that relate to emotional intelligence with the interpersonal skills of the project manager and success in project management. For this, we made a systematic review of literature on the subject. With the results, we provide theoretical basis for the formulation of hypotheses that may be tested in empirical studies.

KEY-WORDS: Emotional intelligence. Project management. Success in Project management. 


\section{INTRODUÇÃO}

A inteligência emocional (IE) é uma temática relativamente recente que amplia o conceito tradicional de inteligência, incluindo aspectos relacionados ao domínio das emoções e dos sentimentos (Woyciekoski \& Hutz, 2009). Com origem no conceito da inteligência social, surgido na década de 1930, o termo IE apareceu pela primeira vez na década de 1980, mas foi somente na década de 1990 que ganhou projeção mundial (Gonzalez, 2012). Salovey e Mayer (1990) definem IE como a habilidade de monitorar sentimentos e emoções próprias e dos outros, e utilizar essas informações para guiar pensamentos e ações. Nas últimas décadas, um grande número de pesquisas tem estudado as habilidades da IE associadas a comportamentos no trabalho, alguns desses estudos apresentaram uma associação significativa entre IE e comportamentos relacionados ao gerenciamento de projetos (Clarke, 2010a; Druskat \& Druskat, 2012; Gonzalez, 2012).

Analisar a IE no contexto de gestão de projetos pode ser relevante uma vez que, segundo Pinto e Rouhiainen (2002), o gerenciamento de projetos envolve de forma natural uma propensão para o surgimento de conflitos, já que as limitações de custo e prazo inerentes a projetos e a complexidade das inter-relações, onde estão presentes os aspectos políticos e organizacionais, são sempre um desafio para o gerente de projetos. Nas últimas décadas, o lado humano do gerente de projetos tem sido identificado como um componente crítico associado ao sucesso no gerenciamento de projeto (Clarke, 2010b). Na última edição de seu guia para gerenciamento de projetos, o PMI (2013) descreve uma lista de habilidades interpessoais que um gerente de projetos deve possuir para gerenciar de forma mais efetiva um projeto, algumas delas são liderança, gerenciamento de conflitos, construção de equipes, comunicação e coaching. Druskat e Druskat (2012) argumentam que, devido ao ambiente de projetos, onde as relações interpessoais se desenvolvem mais rapidamente e ocorrem frequentemente entre diferentes culturas organizacionais, profissionais e pessoais, a necessidade de uma IE acurada é ainda mais acentuada. 
Frame (1999) sugere que o gerenciamento de projetos é formado por três tipos de competências: as individuais, as de equipe e as da organização. O sucesso no gerenciamento de projetos requer competências técnicas e interpessoais, para compreender as situações e as pessoas, e manter um comportamento adequado para o ambiente (Pant \& Baroudi, 2008). De acordo com Dvir, Sadeh \& Malach-Pines (2006), os traços de personalidade do gerente de projetos devem ser adequados ao perfil do projeto para alcançar o sucesso em seu gerenciamento. Os aspectos relacionados às pessoas envolvidas no projeto vêm ganhando importância e sendo foco de um interesse cada vez maior, enfatizando os fatores da personalidade do gerente de projetos e seu estilo de liderança (Carvalho \& Rabechini Jr., 2011). Outro fator que está atraindo a atenção da comunidade científica é o interesse em descobrir como o desenvolvimento da IE contribui na elevação das habilidades do gerente de projeto (Clarke, 2010a).

Além dos aspectos de ordem prática, também é possível perceber a importância de estudos sobre a IE em gestão de projetos do ponto de vista teórico. Hanisch e Wald (2011) dividem a gestão de projetos em dimensões. Para eles existem algumas áreas em que as pesquisas de gerenciamento de projetos estão concentradas e outras em que ainda existem várias lacunas a serem preenchidas. Aliado a isso, no trabalho desenvolvido, Clarke (2010a) sugere que a IE pode ser uma nova área de estudos com potencial preditivo no que tange ao conjunto de comportamentos do gerente de projetos associados ao sucesso no gerenciamento de projetos.

Dessa forma, neste artigo o objetivo é, por meio de uma revisão sistemática da literatura, levantar proposições teóricas que demonstrem a relação entre $I E$, competências interpessoais do gerente de projetos e sucesso no gerenciamento de projetos.

\section{REVISÃO TEÓRICA}

Como base para este trabalho, na fundamentação teórica buscaramse na literatura elementos sobre inteligência emocional, habilidades interpessoais dos gerentes de projetos e sucesso no gerenciamento de 
projetos, de modo a dar o suporte necessário ao objetivo proposto. A seguir esses três pontos são apresentados.

\subsection{INTELIGÊNCIA EMOCIONAL}

A inteligência já foi definida de diferentes formas em diferentes épocas (Salovey \& Mayer, 1990). Ainda segundo Salovey e Mayer (1990), uma das definições de inteligência mais citadas é a de Wechsler (1958), que define inteligência como a capacidade do indivíduo para agir intencionalmente, pensar racionalmente e lidar de forma eficaz com 0 ambiente em que está inserido. A ideia de que existem diferentes tipos de inteligência vem sendo estudada há tempos, uma destas inteligências é a social, definida como a habilidade de entender e gerenciar pessoas (Thorndike \& Stein, 1937). Desde a definição de inteligência social, estudos vêm sendo realizados nessa área, destaca-se o do professor Howard Gardner, da Universidade de Harvard, que, com o livro The shattered mind, introduziu o conceito de inteligências múltiplas. No entanto, o termo IE só veio a aparecer pela primeira vez em 1985 na tese de doutorado de Wayne Payne (Gonzalez, 2012). A IE foi definida academicamente pela primeira vez por Salovey e Mayer (1990), mas, só em 1996, Daniel Goleman popularizou o termo em todo o mundo com seu livro Emotional intelligence (Woyciekoski \& Hutz, 2009).

Salovey e Mayer (1990) definem a IE como um subgrupo da inteligência social, a habilidade de monitorar os sentimentos e emoções próprios e de outras pessoas e utilizar essas informações para guiar pensamentos e ações. Mayer, DiPaolo e Salovey (1990) complementam essa definição dizendo que a IE envolve a avaliação criteriosa e a expressão das emoções em si e nos outros, de forma a melhorar a vida. Para Goleman (1995), IE é a capacidade de motivar a si próprio e de perseguir um objetivo apesar dos percalços, controlando os impulsos e sabendo aguardar a satisfação de seus desejos, mantendo-se em bom estado de espírito e impedindo que a ansiedade interfira na capacidade de raciocinar, de ser empático e autoconfiante. Outra definição para IE é de um conjunto de capacidades não cognitivas, competências e habilidades que influenciam 
como as pessoas lidarão com as demandas e as pressões do dia a dia de forma a alcançar o sucesso (Bar-On, 1997).

Desde o modelo inicial de IE de Salovey \& Mayer (1990), um grande número de modelos para IE foram desenvolvidos (Bar-On, 1997; Cooper \& Sawaf, 1997; Mayer \& Salovey, 1997; Goleman, 1998; Palmer, Stough, Harmer \& Gignac, 2009). Em seu primeiro modelo, Salovey e Mayer (1990) propuseram que a IE era composta por avaliação e expressão das emoções, regulação das emoções e utilização das emoções. Posteriormente, esse modelo foi revisado por Mayer e Salovey (1997) para percepção, avaliação e expressão das emoções, facilitação emocional do pensamento, entendimento e análise das emoções e reflexão e regulação das emoções. No modelo de Goleman (1998), a IE é formada por autopercepção, autorregulação, motivação, empatia e aptidões sociais. Bar-On (1997) em seu modelo inclui novas dimensões. Para ele, a IE é formada por autoconsciência emocional, autoestima, assertividade, autorrealização, independência, empatia, relacionamento interpessoal, responsabilidade social, solução de problemas, teste de realidade, flexibilidade, tolerância ao stress, controle de impulsos, felicidade e otimismo. Mais recentemente Palmer et al. (2009) apresentaram um modelo para IE formado por autoconsciência emocional, expressão emocional, consciência emocional dos outros, raciocínio emocional, autogerenciamento emocional, gerenciamento emocional dos outros e autocontrole emocional.

\subsection{HABILIDADES INTERPESSOAIS DO GERENTE DE PROJETOS}

De acordo com o PMI (2013), gerentes de projetos eficazes precisam adquirir um equilíbrio entre as competências técnicas, interpessoais e conceituais, que os ajudem a analisar as situações e a interagir de forma apropriada. As competências interpessoais são aqueles comportamentos, atitudes e características de personalidade fundamentais, que contribuem para a capacidade de uma pessoa para gerenciar projetos (PMI, 2007). De acordo com El-Sabaa (2001), as habilidades interpessoais do gerente de projetos são demonstradas pela forma como ele percebe e 
reconhece as atitudes de seus superiores, pares e subordinados e a maneira como ele reage a elas.

Para o IPMA (2012), os elementos que compõem as habilidades interpessoais para o gerenciamento de projetos são liderança, comprometimento e motivação, autocontrole, assertividade, descontração, abertura, criatividade, orientação para resultados, eficiência, aconselhamento, negociação, conflitos e crises, confiabilidade, valores e ética. No PMI (2013), descrevem-se liderança, construção de equipes, motivação, comunicação, influência, tomada de decisões, consciência política e cultural, negociação, construção de confiança, gerenciamento de conflitos e coaching como habilidades interpessoais do gerente de projetos. $\mathrm{Na}$ lista de habilidades do gerente de projetos de Kerzner (2009), as competências interpessoais que aparecem são construção de equipes, liderança e resolução de conflitos. Gerentes de projetos que possuem as habilidades listadas apresentam as características necessárias para gerenciar projetos de forma mais eficaz (Alam, Gale, Brown \& Khan, 2010; IPMA, 2012; Kerzner, 2009; PMI, 2013).

\subsection{SUCESSO NO GERENCIAMENTO DE PROJETOS}

O sucesso em projetos é um tema que gera muita controvérsia, não sendo tarefa fácil defini-lo; isso acontece porque ele depende do ponto de vista de análise, assim, diferentes stakeholders terão diferentes opiniões sobre o sucesso de um projeto (Carvalho \& Rabechini Jr., 2011). De acordo com Baccarini (1999), o sucesso do projeto depende de dois componentes distintos - o primeiro é o sucesso do produto do projeto, que está relacionado ao produto final do projeto e dependerá da ótica de análise dos stakeholders; o segundo é o sucesso no gerenciamento de projeto, que tem foco nos processos, em particular no cumprimento das metas de custo, prazo e qualidade, levando-se em consideração a forma como o projeto é conduzido. Dessa forma, o produto de um projeto poderá ser um sucesso, mesmo com uma gestão ineficaz, mas o inverso também é verdadeiro: o gerenciamento de um projeto pode ser um sucesso e mesmo assim o produto do projeto ser um fracasso (De Wit, 1988). 
Neste artigo, tratar-se-á apenas do sucesso no gerenciamento do projeto, que compreende a primeira dimensão do sucesso em projetos do trabalho apresentado por Shenhar, Levy \& Dvir (1997). De acordo com esses autores, essa dimensão mede o quão eficiente foi o gerenciamento do projeto por meio do atendimento ao prazo e ao custo, podendo ser avaliada durante e logo após o término do projeto. Apesar de não indicar sucesso no projeto, o sucesso no gerenciamento do projeto pode ser considerado crítico, pois no ambiente competitivo vivido pelas organizações atualmente, onde os ciclos de vida dos produtos são menores, o time-to-market é um fator crítico de competitividade.

\section{MÉTODO}

A abordagem metodológica utilizada neste trabalho foi revisão sistemática da literatura. De acordo com Green (2005), essa é uma ferramenta científica que pode ser utilizada para avaliar, resumir e comunicar os resultados e implicações de inúmeras pesquisas. Esse é um método de mapeamento de áreas de incerteza, em que se pode identificar que pouca ou nenhuma pesquisa relevante foi realizada, criando lacunas para novos estudos (Petticrew \& Roberts, 2006).

As buscas de artigos foram realizadas nas bases de dados Web of Science e EBSCO, escolhidas pela relevância na comunidade acadêmica, amplitude e pelas opções de tratamento de resultados da busca que oferecem, favorecendo uma análise mais sistemática do tema. Uma busca foi realizada nas duas bases de dados utilizando os seguintes termos "Emotional Intelligence" and "Project Manage*". Na base de dados Web of Science, foram encontrados 30 artigos. Na base de dados EBSCO, após a busca, foram aplicados os filtros "Revistas Acadêmicas", "Journals" e "Duplicatas exatas removidas", o que resultou em 32 artigos. Os resultados das duas buscas foram comparados e encontraram-se 13 artigos que se repetiam, dessa forma, o resultado total de artigos encontrados nas duas bases de dados foi de 49 artigos.

Na etapa seguinte, foi realizada uma análise de cada um dos 49 artigos por meio de seus resumos: aqueles que não estavam alinhados com 
o tema deste trabalho, pois, apesar de conter as palavras-chave, não tratavam de IE nem de gestão de projetos, foram excluídos. Nessa etapa de análise, 20 artigos foram desconsiderados, restando assim um total de 29 artigos, todos abordando e relacionando de alguma forma IE e gestão de projetos. Desses 29 artigos, em três deles não estava disponível o texto integral, por isso também foram desconsiderados. Com isso o número total de artigos ficou em 26, cuja leitura foi realizada na íntegra. Após a avaliação do conteúdo dos artigos, seis deles foram rejeitados devido a sua fragilidade metodológica ou ao não alinhamento com o objetivo deste estudo. Resultaram finalmente 20 artigos o número de trabalhos considerados para elaboração deste estudo.

Com a definição dos artigos, iniciou-se a análise de cada um deles. Na Figura 1, apresenta-se de forma resumida o fluxo metodológico utilizado neste trabalho. Espera-se, dessa forma, contribuir para a teoria, provendo proposições que possam ser utilizados em pesquisas empíricas futuras.

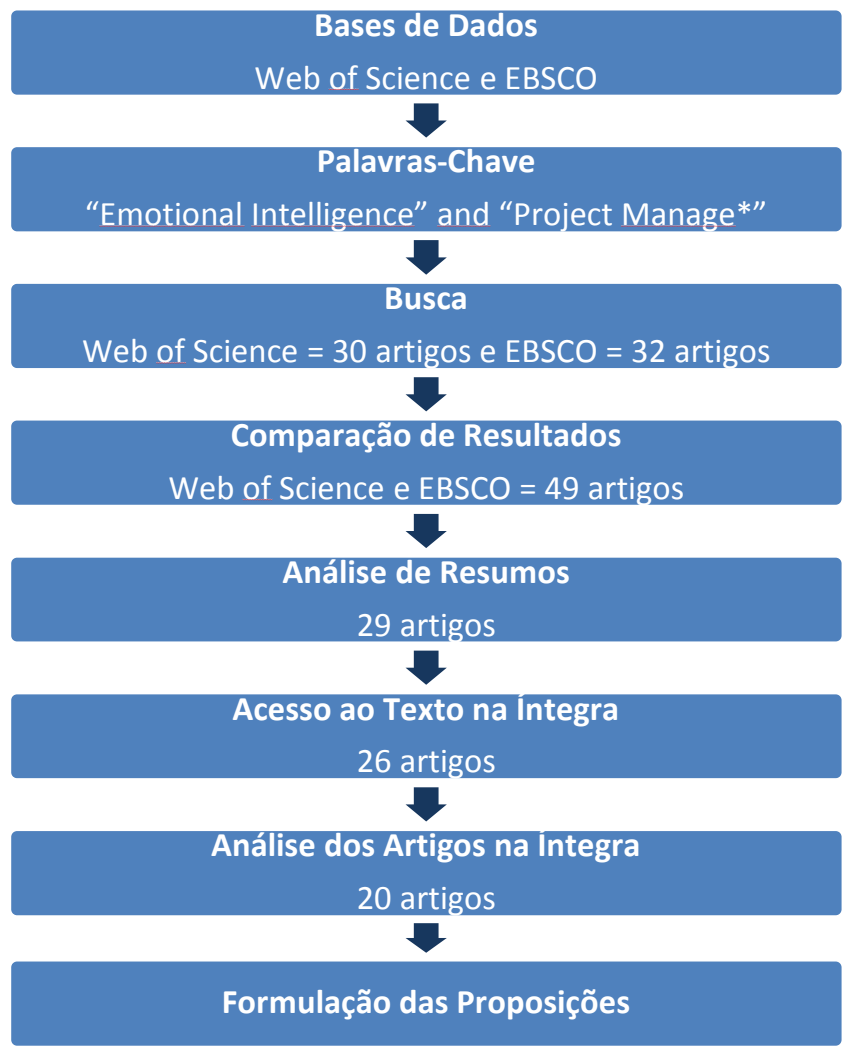

Figura 1: Fluxo Metodológico

Fonte: Elaborado pelos autores 


\section{ANÁLISE DOS RESULTADOS}

Apesar de estudos que relacionem IE a uma serie de comportamentos ligados ao ambiente de trabalho estarem sendo construídos nas últimas décadas (Clarke, 2010a), dentre os artigos selecionados para este trabalho o mais antigo foi elaborado em meados dos anos 2000, enquanto a maioria foi desenvolvida a partir de 2010. Outro ponto relevante é a origem dos estudos, em sua maioria provenientes Europa. A metodologia abordada também tem predominância qualitativa. Os trabalhos de forma geral procuram estudar a relação entre IE e as competências interpessoais dos gerentes de projetos, o sucesso dos projetos e o sucesso dos gerentes de projeto. No Quadro 1, apresenta-se uma síntese dos artigos analisados neste estudo. 


\begin{tabular}{|c|c|c|c|c|c|}
\hline & Autores & Objetivo & Metodologia & $\begin{array}{c}\text { Resultados e } \\
\text { Conclusões }\end{array}$ & Origem \\
\hline 1 & $\begin{array}{l}\text { Turner e } \\
\text { Müller (2005) }\end{array}$ & $\begin{array}{l}\text { Identificar se as } \\
\text { competências do } \\
\text { gerente de projetos, } \\
\text { incluindo } \\
\text { personalidade e } \\
\text { estilos de liderança, } \\
\text { são fatores críticos } \\
\text { de sucesso do } \\
\text { projeto. }\end{array}$ & $\begin{array}{l}\text { Revisão } \\
\text { sistemática da } \\
\text { literatura. }\end{array}$ & $\begin{array}{l}\text { A literatura } \\
\text { encontrada não } \\
\text { apresenta estudos } \\
\text { relevantes do impacto } \\
\text { do gerente de } \\
\text { projetos. Algumas } \\
\text { alternativas, como o } \\
\text { nível da IE do gerente } \\
\text { de projetos que } \\
\text { influencia na } \\
\text { percepção de sucesso } \\
\text { do projeto, podem } \\
\text { explicar a falta de } \\
\text { estudos que } \\
\text { apresentam essa } \\
\text { correlação. No } \\
\text { entanto somente } \\
\text { estudos empíricos } \\
\text { podem comprovar ou } \\
\text { não a sua existência. } \\
\end{array}$ & Europa \\
\hline 2 & $\begin{array}{l}\text { Barry e Plessis } \\
(2007)\end{array}$ & $\begin{array}{l}\text { Discutir se a IE } \\
\text { aplicada ao gerente } \\
\text { de projetos e se } \\
\text { gerentes de projetos } \\
\text { e stakeholders têm a } \\
\text { visão da importância } \\
\text { da IE para o sucesso } \\
\text { do projeto. }\end{array}$ & $\begin{array}{l}\text { Pesquisa } \\
\text { quantitativa com } \\
395 \text { gerentes de } \\
\text { projetos e } \\
\text { stakeholders. }\end{array}$ & $\begin{array}{l}\text { Os resultados da } \\
\text { pesquisa mostraram } \\
\text { que a IE é percebida } \\
\text { como uma importante } \\
\text { competência do } \\
\text { gerente de projeto, } \\
\text { tanto por gerentes de } \\
\text { projetos como por } \\
\text { stakeholders. } \\
\end{array}$ & África do Sul \\
\hline 3 & $\begin{array}{l}\text { Sunindijo, } \\
\text { Hadikusumo e } \\
\text { Ogunlana } \\
(2007)\end{array}$ & $\begin{array}{l}\text { Investigar os } \\
\text { benefícios da IE no } \\
\text { gerenciamento de } \\
\text { projetos no contexto } \\
\text { de estilos efetivos de } \\
\text { liderança. }\end{array}$ & $\begin{array}{l}\text { Pesquisa } \\
\text { quantitativa com } \\
34 \text { gerentes de } \\
\text { projetos e } 12 \\
\text { engenheiros. }\end{array}$ & $\begin{array}{l}\text { Gerentes de projetos } \\
\text { com maior nível de IE } \\
\text { tendem a utilizar uma } \\
\text { comunicação mais } \\
\text { aberta e estilos de } \\
\text { liderança mais } \\
\text { proativos. }\end{array}$ & Tailândia \\
\hline 4 & $\begin{array}{l}\text { Geoghegan e } \\
\text { Dulewicz } \\
(2008)\end{array}$ & $\begin{array}{l}\text { Explorar a existência } \\
\text { de uma relação } \\
\text { estatisticamente } \\
\text { significativa entre as } \\
\text { competências de } \\
\text { liderança do gerente } \\
\text { de projetos e o } \\
\text { sucesso do projeto. }\end{array}$ & $\begin{array}{l}\text { Pesquisa } \\
\text { quantitativa com } \\
52 \text { gerentes de } \\
\text { projetos de uma } \\
\text { empresa líder em } \\
\text { serviços } \\
\text { financeiros no } \\
\text { Reino Unido. }\end{array}$ & $\begin{array}{l}\text { Os resultados } \\
\text { demonstram uma } \\
\text { correlação significante } \\
\text { entre o sucesso do } \\
\text { projeto e as } \\
\text { competências de } \\
\text { liderança do gerente } \\
\text { de projetos. Também } \\
\text { foi possível verificar } \\
\text { que gerentes de } \\
\text { projetos com nível } \\
\text { elevado de IE } \\
\text { possuem melhor } \\
\text { performance de } \\
\text { liderança. }\end{array}$ & Europa \\
\hline 5 & $\begin{array}{l}\text { Clarke } \\
(2010 \mathrm{~b})\end{array}$ & $\begin{array}{l}\text { Examinar os efeitos } \\
\text { de treinamento sobre } \\
\text { IE para gerentes de } \\
\text { projetos. }\end{array}$ & $\begin{array}{l}\text { Pesquisa } \\
\text { quantitativa com } \\
53 \text { gerentes de } \\
\text { projeto do Reino } \\
\text { Unido. }\end{array}$ & $\begin{array}{l}\text { O estudo mostrou que } \\
\text { os resultados de um } \\
\text { treinamento em IE só } \\
\text { aparecem de forma } \\
\text { relevante seis meses } \\
\text { após o treinamento. }\end{array}$ & Europa \\
\hline
\end{tabular}

Continua 


\begin{tabular}{|c|c|c|c|c|c|}
\hline \multicolumn{6}{|c|}{ Continuação } \\
\hline & Autores & Objetivo & Metodologia & $\begin{array}{l}\text { Resultados e } \\
\text { Conclusões }\end{array}$ & Origem \\
\hline 6 & $\begin{array}{l}\text { Suhonen e } \\
\text { Paasivaara } \\
(2010)\end{array}$ & $\begin{array}{l}\text { Explorar as } \\
\text { dimensões e o } \\
\text { conteúdo dos estudos } \\
\text { sobre o capital } \\
\text { humano em } \\
\text { gerenciamento de } \\
\text { projetos. }\end{array}$ & $\begin{array}{l}\text { Revisão } \\
\text { sistemática da } \\
\text { literatura. }\end{array}$ & \begin{tabular}{|l} 
Existem poucos \\
estudos sobre o \\
capital humano em \\
gerenciamento de \\
projetos. A IE no \\
contexto de \\
gerenciamento de \\
projetos não pode ser \\
subestimada. É \\
possível elevar o nível \\
de IE dos gerentes de \\
projeto.
\end{tabular} & Europa \\
\hline 7 & $\begin{array}{l}\text { Müller e } \\
\text { Turner } \\
(2010 a)\end{array}$ & $\begin{array}{l}\text { Identificar o impacto } \\
\text { da personalidade do } \\
\text { gerente de projetos } \\
\text { no sucesso do } \\
\text { projeto. }\end{array}$ & $\begin{array}{l}\text { Pesquisa } \\
\text { quantitativa com } \\
400 \text { gerentes de } \\
\text { projetos de } \\
\text { diversos países. }\end{array}$ & $\begin{array}{l}\text { O sucesso do } \\
\text { gerenciamento de } \\
\text { projeto está mais } \\
\text { fortemente } \\
\text { relacionado às } \\
\text { competências e às } \\
\text { atitudes de liderança } \\
\text { do gerente de } \\
\text { projetos, enquanto o } \\
\text { sucesso do projeto } \\
\text { está relacionado a } \\
\text { uma combinação } \\
\text { entre as atitudes e } \\
\text { competências de } \\
\text { liderança e a IE do } \\
\text { gerente de projetos. } \\
\end{array}$ & Europa \\
\hline 8 & $\begin{array}{l}\text { Clarke } \\
(2010 a)\end{array}$ & $\begin{array}{l}\text { Examinar a relação } \\
\text { entre IE, as } \\
\text { competências do } \\
\text { gerenciamento de } \\
\text { projetos e a liderança } \\
\text { transformacional. }\end{array}$ & $\begin{array}{l}\text { Pesquisa } \\
\text { quantitativa com } \\
67 \text { gerentes de } \\
\text { projetos de duas } \\
\text { organizações do } \\
\text { Reino Unido. }\end{array}$ & $\begin{array}{l}\text { Foi encontrada uma } \\
\text { ênfase na relação } \\
\text { entre a IE e } \\
\text { comportamentos dos } \\
\text { gerentes de projetos } \\
\text { associados e trabalho } \\
\text { em equipe, } \\
\text { comunicação e gestão } \\
\text { de conflitos e com o } \\
\text { sucesso dos } \\
\text { resultados do projeto. }\end{array}$ & Europa \\
\hline 9 & $\begin{array}{l}\text { Müller e } \\
\text { Turner } \\
(2010 b)\end{array}$ & $\begin{array}{l}\text { Identificar os perfis } \\
\text { de liderança de } \\
\text { gerentes de projetos } \\
\text { de sucesso. }\end{array}$ & $\begin{array}{l}\text { Pesquisa } \\
\text { quantitativa com } \\
400 \text { gerentes de } \\
\text { projetos de } \\
\text { diversos países. }\end{array}$ & $\begin{array}{l}\text { Os resultados } \\
\text { indicaram que } \\
\text { gerentes de projeto } \\
\text { de sucesso têm um } \\
\text { nível elevado em três } \\
\text { subdimensões da IE } \\
\text { (influência, motivação } \\
\text { e conscienciosidade). }\end{array}$ & Europa \\
\hline 10 & Davis (2011) & $\begin{array}{l}\text { Determinar a } \\
\text { correlação entre um } \\
\text { modelo de medida da } \\
\text { IE e as competências } \\
\text { interpessoais do } \\
\text { gerente de projetos. }\end{array}$ & $\begin{array}{l}\text { Observação quase } \\
\text { experimental com } \\
\text { métodos } \\
\text { qualitativos e } \\
\text { quantitativos. }\end{array}$ & $\begin{array}{l}\text { Os resultados } \\
\text { mostraram uma } \\
\text { relação } \\
\text { estatisticamente } \\
\text { significante entre o } \\
\text { modelo de IE utilizado } \\
\text { e as competências } \\
\text { interpessoais do } \\
\text { gerente de projetos. } \\
\end{array}$ & Estados Unidos \\
\hline
\end{tabular}

Continua 
Continuação

\begin{tabular}{|c|c|c|c|c|c|}
\hline & Autores & Objetivo & Metodologia & \begin{tabular}{|l|} 
Resultados e \\
Conclusões \\
\end{tabular} & Origem \\
\hline 11 & $\begin{array}{l}\text { Müller, Geraldi } \\
\text { e Turner } \\
(2012)\end{array}$ & $\begin{array}{l}\text { Estudar como a } \\
\text { complexidade do } \\
\text { projeto influencia na } \\
\text { relação entre as } \\
\text { competências de } \\
\text { liderança do gerente } \\
\text { de projeto e o } \\
\text { sucesso do projeto. }\end{array}$ & $\begin{array}{l}\text { Pesquisa } \\
\text { quantitativa com } \\
136 \text { gerentes de } \\
\text { projeto, em sua } \\
\text { maioria da } \\
\text { América do Norte. }\end{array}$ & $\begin{array}{l}\text { A relação entre IE e o } \\
\text { sucesso do projeto é } \\
\text { moderada pela } \\
\text { complexidade do } \\
\text { projeto. O aumento } \\
\text { da IE impacta } \\
\text { diretamente e } \\
\text { positivamente no } \\
\text { sucesso do projeto. }\end{array}$ & Europa \\
\hline 12 & $\begin{array}{l}\text { Obradovic, } \\
\text { Jovanovic, } \\
\text { Petrovic, Mihic } \\
\text { e Mitrovic } \\
\text { (2013) }\end{array}$ & $\begin{array}{l}\text { Estudar a correlação } \\
\text { entre a IE dos } \\
\text { gerentes de projeto, } \\
\text { seu sucesso } \\
\text { profissional e seu } \\
\text { nível de graduação. } \\
\end{array}$ & $\begin{array}{l}\text { Pesquisa } \\
\text { quantitativa com } \\
75 \text { gerentes de } \\
\text { projeto da Sérvia. }\end{array}$ & $\begin{array}{l}\text { Os resultados } \\
\text { sugerem que, quanto } \\
\text { maior o nível de IE do } \\
\text { gerente de projetos, } \\
\text { maior é o seu sucesso } \\
\text { profissional. } \\
\end{array}$ & Europa \\
\hline 13 & $\begin{array}{l}\text { Zhang, Zuo e } \\
\text { Zillante (2013) }\end{array}$ & $\begin{array}{l}\text { Identificar e avaliar } \\
\text { as competências } \\
\text { emocionais chaves } \\
\text { do gerente de projeto } \\
\text { de indústrias de } \\
\text { construção chinesas. }\end{array}$ & $\begin{array}{l}\text { Pesquisa } \\
\text { quantitativa com } \\
275 \text { gerentes de } \\
\text { projeto chineses. }\end{array}$ & $\begin{array}{l}\text { Os resultados } \\
\text { mostraram que } \\
\text { existem algumas } \\
\text { competências } \\
\text { emocionais } \\
\text { importantes para os } \\
\text { gerentes de projetos } \\
\text { de indústrias de } \\
\text { construção chinesas. } \\
\end{array}$ & China \\
\hline 14 & $\begin{array}{l}\text { Lindebaum e } \\
\text { Jordan (2012) }\end{array}$ & $\begin{array}{l}\text { Como a IE dos } \\
\text { gerentes de projetos } \\
\text { se relaciona com sua } \\
\text { performance em } \\
\text { projetos de } \\
\text { construção. }\end{array}$ & $\begin{array}{l}\text { Pesquisa } \\
\text { quantitativa com } \\
55 \text { gerentes de } \\
\text { projetos de } \\
\text { indústrias de } \\
\text { construção do } \\
\text { Reino Unido. }\end{array}$ & $\begin{array}{l}\text { A IE não contribui } \\
\text { para todos os } \\
\text { aspectos de } \\
\text { performance de } \\
\text { projeto. Ela pode não } \\
\text { contribuir para a } \\
\text { melhora da } \\
\text { performance dos } \\
\text { projetos. }\end{array}$ & Europa \\
\hline 15 & $\begin{array}{l}\text { Lindebaum e } \\
\text { Cassel (2012) }\end{array}$ & $\begin{array}{l}\text { Explorar a natureza } \\
\text { da IE aplicada a } \\
\text { projetos de } \\
\text { construção. }\end{array}$ & $\begin{array}{l}\text { Pesquisa } \\
\text { qualitativa com } \\
\text { gerentes de } \\
\text { projetos do Reino } \\
\text { Unido. } \\
\end{array}$ & $\begin{array}{l}\text { A IE tem um papel } \\
\text { relevante no contexto } \\
\text { das indústrias de } \\
\text { construção. }\end{array}$ & Europa \\
\hline 16 & $\begin{array}{l}\text { Trivellas e } \\
\text { Drimoussis } \\
(2013)\end{array}$ & $\begin{array}{l}\text { Investigar a relação } \\
\text { do perfil de } \\
\text { competências } \\
\text { interpessoais do } \\
\text { gerente de projeto e } \\
\text { o sucesso do projeto } \\
\text { no contexto grego. } \\
\end{array}$ & $\begin{array}{l}\text { Pesquisa } \\
\text { quantitativa com } \\
97 \text { gerentes de } \\
\text { projetos da } \\
\text { Grécia. }\end{array}$ & $\begin{array}{l}\text { Os resultados } \\
\text { mostraram que } \\
\text { projetos com gerente } \\
\text { de projeto com maior } \\
\text { nível de IE possuem } \\
\text { melhores resultados. }\end{array}$ & Europa \\
\hline 17 & $\begin{array}{l}\text { Zhang e Fan } \\
(2013)\end{array}$ & $\begin{array}{l}\text { Identificar a } \\
\text { correlação entre a IE } \\
\text { dos gerentes de } \\
\text { projeto e a } \\
\text { performance dos } \\
\text { projetos de } \\
\text { construção. }\end{array}$ & $\begin{array}{l}\text { Pesquisa } \\
\text { quantitativa com } \\
112 \text { gerentes de } \\
\text { projetos chineses } \\
\text { da área de } \\
\text { construção. }\end{array}$ & $\begin{array}{l}\text { Uma forte correlação } \\
\text { positiva foi } \\
\text { encontrada entre o } \\
\text { nível de IE dos } \\
\text { gerentes de projetos } \\
\text { e a performance de } \\
\text { projetos de } \\
\text { construção. Cada } \\
\text { dimensão da IE afeta } \\
\text { a performance dos } \\
\text { projetos em } \\
\text { diferentes níveis. } \\
\end{array}$ & China \\
\hline
\end{tabular}

Continua 


\begin{tabular}{|c|c|c|c|c|c|}
\hline \multicolumn{6}{|c|}{ Eontinuação } \\
\hline & Autores & Objetivo & Metodologia & $\begin{array}{l}\text { Resultados e } \\
\text { Conclusões }\end{array}$ & Origem \\
\hline 18 & $\begin{array}{l}\text { Lee, Park e } \\
\text { Lee (2013) }\end{array}$ & $\begin{array}{l}\text { Explorar a relação } \\
\text { entre as } \\
\text { competências de } \\
\text { liderança dos } \\
\text { gerentes de projetos } \\
\text { e a performance dos } \\
\text { projetos. }\end{array}$ & $\begin{array}{l}\text { Pesquisa } \\
\text { quantitativa com } \\
298 \text { participantes. }\end{array}$ & $\begin{array}{l}\text { A IE tem influência } \\
\text { direta no desempenho } \\
\text { do projeto em todos } \\
\text { os momentos, } \\
\text { independentemente } \\
\text { do tempo do projeto. }\end{array}$ & Coreia do Sul \\
\hline 19 & $\begin{array}{l}\text { Mazur, } \\
\text { Pisarski, } \\
\text { Chang e } \\
\text { Ashkanasy } \\
(2014)\end{array}$ & $\begin{array}{l}\text { Explorar como a } \\
\text { relação entre } \\
\text { stakeholders e os } \\
\text { principais gerentes } \\
\text { de projetos da } \\
\text { Austrália influenciam } \\
\text { no sucesso do } \\
\text { projeto. }\end{array}$ & $\begin{array}{l}\text { Pesquisa } \\
\text { quantitativa com } \\
313 \text { gerentes de } \\
\text { projeto da } \\
\text { Austrália. }\end{array}$ & $\begin{array}{l}\text { Os resultados } \\
\text { encontrados suportam } \\
\text { que a IE dos gerentes } \\
\text { de projeto está } \\
\text { positivamente } \\
\text { relacionada com uma } \\
\text { comunicação efetiva e } \\
\text { de qualidade com } \\
\text { stakeholders internos } \\
\text { e externos do projeto. } \\
\text { Esse tipo de } \\
\text { relacionamento } \\
\text { aumenta as chances } \\
\text { de sucesso do } \\
\text { projeto. }\end{array}$ & Austrália \\
\hline 20 & Trejo (2014) & $\begin{array}{l}\text { Identificar a relação } \\
\text { entre a IE de } \\
\text { trabalhadores } \\
\text { hispânicos nos } \\
\text { resultados dos } \\
\text { projetos em que eles } \\
\text { trabalham. }\end{array}$ & $\begin{array}{l}\text { Pesquisa } \\
\text { quantitativa com } \\
88 \text { integrantes de } \\
\text { projetos de } \\
\text { origem hispânica. }\end{array}$ & $\begin{array}{l}\text { Os resultados } \\
\text { mostram que a IE dos } \\
\text { membros de equipe } \\
\text { estão positivamente } \\
\text { correlacionadas com } \\
\text { os resultados dos } \\
\text { projetos; as } \\
\text { dimensões da IE } \\
\text { relacionam-se em } \\
\text { diferentes níveis com } \\
\text { os resultados do } \\
\text { projeto. }\end{array}$ & Estados Unidos \\
\hline
\end{tabular}

\section{Quadro 1: Síntese dos artigos analisados}

Fonte: Elaborado pelos autores

\section{CONSIDERAÇÕES FINAIS}

\subsection{FORMULAÇÃO DAS PROPOSIÇÕES}

Dentre os artigos analisados neste trabalho, nove apresentam estudos que relacionam a IE ao perfil de liderança do gerente de projetos (Clarke, 2010a; Geoghegan \& Dulewicz, 2008; Lee et al., 2013; Müller et al., 2012; Müller \& Turner, 2010a, 2010b; Sunindijo et al., 2007; Trivellas \& Drimoussis, 2013; Turner \& Müller, 2005). Esses artigos especificam uma lista de competências ou dimensões de liderança, necessárias para os gerentes de projetos. A IE, de acordo com esses artigos, é formada por um 
conjunto dessas competências ou dimensões. Os autores sugerem que a IE tem correlação com as demais competências do gerente de projeto. No estudo, Clarke (2010a) mostra a relação entre IE e as competências de comunicação, trabalho em equipe, atenção e gerenciamento de conflitos. No trabalho apresentado, Davis (2011) segue a mesma linha de Clarke (2010a), relacionando ainda a capacidade de motivação e resolução de problemas. Com os resultados apresentados por esses artigos surge a primeira proposição:

\section{Proposição 1: A Inteligência Emocional tem uma relação positiva com as competências interpessoais do gerente de projetos.}

Utilizando outra abordagem, em três artigos relaciona-se a IE ao sucesso do gerente de projetos (Müller \& Turner, 2010b; Obradovic et al., 2013; Trivellas \& Drimoussis, 2013). De acordo com os resultados encontrados por Obradovic et al. (2013), os gerentes de projetos com maior nível de IE são mais bem sucedidos profissionalmente. Esse resultado corrobora os achados de Trivellas e Drimoussis (2013), que adicionalmente demonstram que as dimensões da IE se relacionam em diferentes níveis com o sucesso profissional do gerente de projeto. Dessa forma, é possível elaborar a seguinte proposição:

\section{Proposição 2: A Inteligência Emocional tem uma relação positiva com o sucesso profissional dos gerentes de projetos.}

Em cinco artigos, os autores sugerem que o conjunto de competências que formam a IE são fatores críticos para o sucesso do projeto (Geoghegan \& Dulewicz, 2008; Mazur et al., 2014; Müller et al., 2012; Müller \& Turner, 2010a; Turner \& Müller, 2005). Por meio de revisão da literatura, Turner e Müller (2005) concluíram que o impacto das competências de liderança do gerente de projetos, o que inclui a IE, no sucesso dos projetos, tem sido ignorada nos estudos sobre os fatores de sucesso dos projetos. Para Müller e Turner (2010a), a IE está diretamente relacionada ao sucesso dos projetos. Como se nota, nesses estudos abordase de forma direta a relação da IE com o sucesso dos projetos, porém a 
relação da IE com o sucesso no gerenciamento de projetos é abordada de forma indireta. Os autores reconhecem que o sucesso do projeto tem uma dimensão maior que o sucesso no gerenciamento do projeto, sendo este último um dos componentes do sucesso do projeto.

Em seis dos artigos analisados, relaciona-se a IE ao gerenciamento de projetos da área de construção (Cacamis \& El Asmar, 2013; Lindebaum \& Cassel, 2012; Lindebaum \& Jordan, 2012; Sunindijo et al., 2007; Zhang et al., 2013; Zhang \& Fan, 2013). Nos estudos apresentados, Cacamis e El Asmar (2013) e Zhang e Fan (2013) apontam para gerentes de projetos com nível elevado de IE como forma de melhorar os resultados dos projetos de construção. Esse número significativo de estudos em projetos de construção reflete a preocupação desse segmento com o tema. Também com foco em uma área específica, Lee et al. (2013) relacionam projetos de TI à IE. Assim como nos trabalhos sobre projetos de construção, os autores sugerem que o nível de IE do gerente de projetos melhora os resultados dos projetos de TI.

A IE também pode ser analisada no contexto da equipe de projetos, Trejo (2014) apresenta essa abordagem. De acordo com o autor, o nível de IE apresentado pela equipe de projeto contribui de forma relevante para o sucesso do projeto. Apesar de tratar de uma população bem específica, em sua pesquisa Trejo (2014) fornece indícios de que equipes com nível elevado de IE trazem melhores resultados aos projetos. Assim, a seguinte proposição pode ser elaborada:

\section{Proposição 3: A Inteligência Emocional das equipes de projetos e do gerente de projetos está diretamente relacionada com o sucesso no gerenciamento do projeto.}

De forma mais genérica, em seus trabalhos Barry e Plessis (2007) e Suhonen e Paasivaara (2010) enfatizam a importância das competências interpessoais para o gerenciamento de projetos. Nesses artigos, os autores demonstram a importância da IE no gerenciamento de projetos, dessa forma, elevar o nível da IE dos gerentes de projetos também é uma forma de contribuir para o bom desempenho do gerenciamento do projeto. Nessa linha, Clarke (2010b) demonstra que é possível por meio de treinamento 
elevar o nível de IE do gerente de projetos, possibilitando a elaboração da última proposição deste artigo:

\section{Proposição 4: Programas específicos de treinamento têm relação positiva com o nível de Inteligência Emocional dos gerentes de projetos.}

A relação entre $I E$, competências e sucesso em gestão de projetos, objeto deste estudo, enseja a transformação das quatro proposições aqui formuladas em hipóteses, representadas no modelo apresentado na Figura 2 , a serem verificadas empiricamente em estudos futuros.

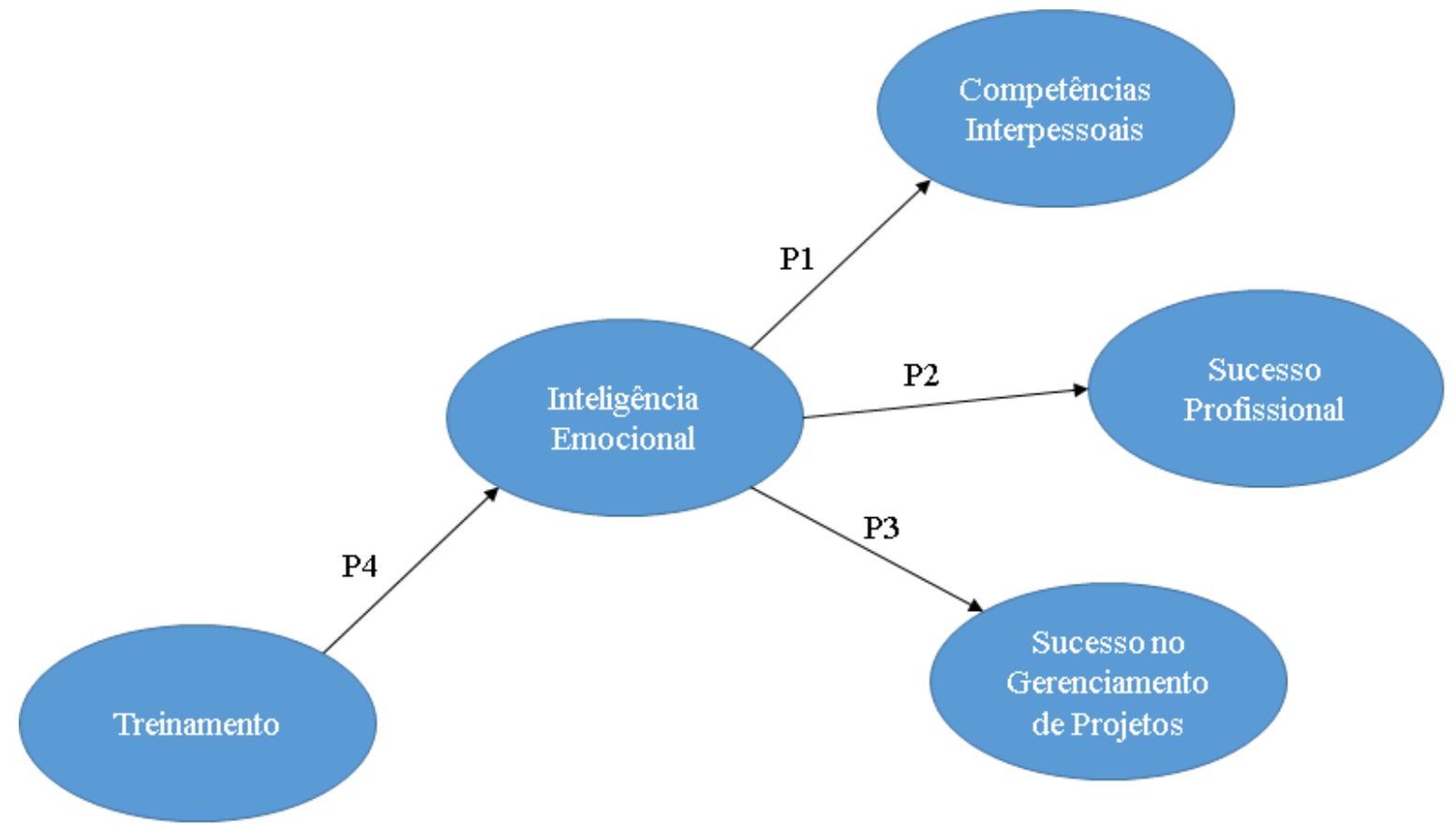

Figura 2: Modelo Proposto

Fonte: Elaborado pelos autores

\subsection{CONSIDERAÇÕES ACADÊMICAS}

A IE, como já mencionado, é um fenômeno relativamente recente (Woyciekoski \& Hutz, 2009) que teve origem na década de 1980 (Gonzalez, 2012); no entanto os resultados encontrados apontam que a associação da IE ao gerenciamento de projetos é ainda mais recente, com estudos a partir de meados dos anos 2000. Nota-se também uma concentração de trabalhos de origem europeia, demonstrando uma lacuna a ser preenchida em relação 
à repetibilidade dos resultados encontrados nesses trabalhos em outras culturas.

Os resultados encontrados também sugerem a IE como um componente relevante das competências interpessoais do gerente de projeto. A correlação entre IE e competências como comunicação, trabalho em equipe, gerenciamento de conflitos, motivação e resolução de problemas encontrada, por exemplo, nos estudos de Clarke (2010a) e Davis (2011) exemplifica essa relevância. No entanto, ainda são poucos os estudos nesse campo, além de concentrados na realidade europeia, abrindo, assim, espaço para a realização de novos estudos que levem em conta outras realidades, além da possível diferença entre os níveis de correlação das dimensões da IE e das competências interpessoais do gerente de projeto.

Em outra abordagem, os estudos analisados apresentam a IE dos gerentes de projetos como um dos fatores críticos de sucesso para o projeto. Corroborando os resultados encontrados Müller e Turner (2010a), demonstram que a IE dos gerentes de projetos complementa e equilibra a lista de fatores críticos para o sucesso do projeto. Seguindo a linha sugerida por Baccarini (1999), em que o sucesso do gerenciamento de projeto é um componente do sucesso do projeto e está ligado ao cumprimento de metas de prazo, custo e qualidade, percebe-se que a maior parte dos trabalhos analisados com esse contexto focam o sucesso do projeto e deixam lacunas no que diz respeito ao sucesso do gerenciamento de projetos.

As proposições formuladas a partir da revisão da literatura visam fornecer elementos para futuros estudos científicos que possam vir a preencher algumas das lacunas sugeridas. Por meio dessas proposições, espera-se o desenvolvimento de hipóteses que possam ser testadas e validadas em pesquisas, confirmando ou não a relevância da IE no contexto do gerenciamento de projeto. Espera-se também confirmar ou não com futuras pesquisas as correlações sugeridas pelos autores entre IE dos gerentes de projeto, suas competências interpessoais e o sucesso no gerenciamento do projeto. 


\section{REFERÊNCIAS}

Alam, M., Gale, A., Brown, M., \& Khan, A. I. (2010). The importance of human skills in project management professional development. International Journal of Managing Projects in Business, 3(3), 495-516.

Baccarini, D. (1999). The logical framework method for defining project success. Project Management Journal, 30(4), 25-32.

Bar-On, R. (1997). Emotional quotient inventory: technical manual. Toronto: Multi-Health Systems.

Barry, M. L., \& Plessis, Y. (2007). Emotional intelligence: a crucial human resource management ability for engineering project managers in the 21st century. Proceedings of the Africon 2007, Windhoek, Namibia.

Cacamis, M. E., \& El Asmar, M. (2013). Improving project performance through partnering and emotional intelligence. Practice Periodical on Structural Design and Construction, 19(1), 50-56.

Carvalho, M. M., \& Rabechini Jr., R. (2011). Fundamentos em gestão de projetos: construindo competências para gerenciar projetos (3a ed.). São Paulo: Atlas.

Clarke, N. (2010a). Emotional intelligence and its relationship to transformational leadership and key project manager competences. Project Management Journal, 41(2), 5-20.

Clarke, N. (2010b). The impact of a training programme designed to target the emotional intelligence abilities of project managers. International Journal of Project Management, 28(5), 461-468.

Cooper, R. K., \& Sawaf, A. (1997). Emotional intelligence in leadership and organizations. New York: The Berkley Publishing Group.

Davis, S. A. (2011). Investigating the impact of project managers' emotional intelligence on their interpersonal competence. Project Management Journal, 42(4), 37-57.

De Wit, A. (1988). Measurement of project success. International Journal of Project Management, 6(3), 164-170.

Druskat, V., \& Druskat, P. (2012). Applying emotional intelligence in project working. In S. Pryke, \& H. Smyth (Eds.), The management of complex projects: a relationship approach (pp. 78-96). Oxford, UK: Blackwell.

Dvir, D., Sadeh, A., \& Malach-Pines, A. (2006). Projects and project managers: the relationship between project managers' personality, project types, and project success. Project Management Journal, 37(5), 36-48. 
El-Sabaa, S. (2001). The skills and career path of an effective project manager. International Journal of Project Management, 19(1), 1-7.

Frame, J. D. (1999). Project management competence:building key skills for individuals, teams and organizations. San Francisco: Jossey-Bass.

Geoghegan, L., \& Dulewicz, V. (2008). Do project managers' leadership competencies contribute to project success? Project Management Journal, $39(4), 58-67$.

Goleman, D. (1995). Inteligência emocional: a teoria revolucionária que redefine o que é ser inteligente (33a ed., M. Santarrita, Trad.). Rio de Janeiro: Objetiva.

Goleman, D. (1998). Working with emotional intelligence. New York: Bantam Books.

Gonzalez, M. D. (2012). The role of emotional intelligence (EI) in project management over the next five years. International Handbook of Academic Research and Teaching, 22, 83-90.

Green, S. (2005). Systematic reviews and meta-analysis. Evidence-based Medicine and Healthcare, 46(6), 270-274.

Hanisch, B., \& Wald, A. (2011). A project management research framework integrating multiple theoretical perspectives and influencing factors. Project Management Journal, 42(3), 4-22.

IPMA. (2012). NCB National Competence Baseline Versão 3.0. Brasil: IPMA Brasil.

Kerzner, H. (2009). Project management: A systems approach to planning, scheduling and controlling (10th ed.). New Jersey: John Wiley \& Sons.

Lee, H., Park, J., \& Lee, J. (2013). Role of leadership competencies and team social capital in IT services. Journal of Computer Information Systems, 53(4), 1-11.

Lindebaum, D., \& Cassel, C. (2012). A contradiction in terms? Making sense of emotional intelligence in a construction management environment. British Journal of Management, 23(1), 65-79.

Lindebaum, D., \& Jordan, P. J. (2012). Relevant but exaggerated: the effects of emotional intelligence on project manager performance in construction. Construction Management and Economics, 30(7), 575-583.

Mayer, J. D., DiPaolo, M., \& Salovey, P. (1990). Perceiving affective content in ambiguous visual stimuli: A component of emotional intelligence. Journal of Personality Assessment, 54(3-4), 772-781.

Mayer, J. D., \& Salovey, P. (1997). What is emotional intelligence? In P. Salovey \& D. J. Sluyter (Eds.), Educational evelopment and emotional intelligence: Educational implications (pp. 3-31). New York: Basic Books. 
Mazur, A., Pisarski, A., Chang, A., \& Ashkanasy, N. M. (2014). Rating defence major project success: The role of personal attributes and stakeholder relationships. International Journal of Project Management, 32(6), 944-957.

Müller, R., Geraldi, J., \& Turner, J. R. (2012). Relationships between leadership and success in different types of project complexities. IEEE Transactions on Engineering Management, 59(1), 77-90.

Müller, R., \& Turner, J. R. (2010a). Attitudes and leadership competences for project success. Baltic Journal of Management, 5(3), 307-329.

Müller, R., \& Turner, J. R. (2010b). Leadership competency profiles of successful project managers. International Journal of Project Management, 28(5), 437-448.

Obradovic, V., Jovanovic, P., Petrovic, D., Mihic, M., \& Mitrovic, Z. (2013). Project managers' emotional intelligence - A ticket to success. Procedia Social and Behavioral Sciences, 74, 274-284.

Palmer, B. R., Stough, C., Harmer, R., \& Gignac, G. (2009). The genos emotional intelligence inventory: A measure designed specifically for workplace applications. In J. D. A. Parker, D. H. Saklofske, \& C. Stough (Eds.), Assessing emotional intelligence (pp. 103-117). New York: Springer. Retrieved from http://link.springer.com/chapter/10.1007/9780-387-88370-0_6

Pant, I., \& Baroudi, B. (2008). Project management education: The human skills imperative. International Journal of Project Management, 26(2), 124-128.

Petticrew, M., \& Roberts, H. (2006). Systematic reviews in the social sciences. Oxford, UK: Blackwell Publishing.

Pinto, J. K., \& Rouhiainen, P. (2002). Building customer-based project . John Wiley \& Sons.

PMI. (2007). Project Manager Competency Development (PMCD) Framework (2nd ed.). Pennsylvania: Project Management Institute, Inc.

PMI. (2013). A Guide to the Project Management Body of Knowledge (5th ed.). Pennsylvania: Project Management Institute, Inc.

Salovey, P., \& Mayer, J. D. (1990). Emotional Intelligence. Imagination, Cognition and Personality, 9(3), 185-211.

Shenhar, A. J., Levy, O., \& Dvir, D. (1997). Mapping the Dimensions of Project Success. Project Management Journal, 28(2), 5-13.

Suhonen, M., \& Paasivaara, L. (2010). Shared human Capital in Project Management: A Systematic Review of the Literature. Project Management Journal, 42(2), 4-16. 
Sunindijo, R. Y., Hadikusumo, B. H. W., \& Ogunlana, S. (2007). Emotional Intelligence and Leadership Styles in Construction Project Management. Journal of Management in Engineering, 23(4), 166-170.

Thorndike, R. L., \& Stein, S. (1937). An Evaluation of the Attempts to Measure Social Intelligence. Psychological Bulletin, 34(5), 275-284.

Tranfield, D., Denyer, D., \& Smart, P. (2003). Towards a Methodology for Developing Evidence-Informed Management Knowledge by Means of Systematic Review. British Journal of Management, 14(3), 207-222.

Trejo, A. (2014). Emotional Intelligence and Project Outcomes in Technology. International Management Review, 10(1), 31-42.

Trivellas, P., \& Drimoussis, C. (2013). Investigating Leadership Styles, Behavioural and Managerial Competency Profiles of Successful Project Managers in Greece. Procedia-Social and Behavioral Sciences, 73, 692700.

Turner, J. R., \& Müller, R. (2005). The Project Manager's Leadership Style as a Success Factor on Projects: A Literature Review. Project Management Journal, 36(1), 49-61.

Wechsler, D. (1958). The Measurement ans Appraisal os Adult Intelligence (4th ed.). Baltimore: The Williams \& Wilkins Company.

Woyciekoski, C., \& Hutz, C. S. (2009). Inteligência Emocional: Teoria, Pesquisa, Medida, Aplicações e Controvérsia. Psicologia: Reflexão E Crítica, 22(1), 1-11.

Zhang, F., Zuo, J., \& Zillante, G. (2013). Identification and avaluation of the key social competencies for Chinese construction project managers. International Journal of Project Management, 31(5), 748-759.

Zhang, L., \& Fan, W. (2013). Improving performance of construction projects: A project manager's emotional intelligence approach. Engineering, Construction and Architectural Management, 20(2), 195207. 